\title{
On differentiability of strongly $\alpha(\cdot)$-paraconvex functions in non-separable Asplund spaces
}

\author{
by
}

\author{
S. Rolewicz (Warszawa)
}

\begin{abstract}
In Rolewicz (2002) it was proved that every strongly $\alpha(\cdot)$-paraconvex function defined on an open convex set in a separable Asplund space is Fréchet differentiable on a residual set. In this paper it is shown that the assumption of separability is not essential.
\end{abstract}

1. Introduction. Properties of $\alpha(\cdot)$-paraconvex functions. Let $(X,\|\cdot\|)$ be a real Banach space. Let $f$ be a real-valued convex continuous function defined on an open convex subset $\Omega \subset X$. Mazur (1933) proved that there is a subset $A_{\mathrm{G}} \subset \Omega$ of the first Baire category such that $f$ is Gateaux differentiable on $\Omega \backslash A_{\mathrm{G}}$. Asplund (1968) showed that if additionally $X$ is an Asplund space (in particular if $X$ has a separable dual), then there is a subset $A_{\mathrm{F}} \subset \Omega$ of the first Baire category such that $f$ is Fréchet differentiable on $\Omega \backslash A_{\mathrm{F}}$.

The result of Asplund was extended in Rolewicz (2002) to a larger class of functions called strongly $\alpha(\cdot)$-paraconvex, under the additional hypothesis that $X$ is a separable Asplund space (i.e. it is a separable space with separable dual).

In the present paper we shall prove it without this additional hypothesis. First we recall the definitions of $\alpha(\cdot)$-paraconvex and strongly $\alpha(\cdot)$ paraconvex functions.

Let $(X,\|\cdot\|)$ be a real Banach space and $f$ be a real-valued function defined on an open convex subset $\Omega \subset X$. Let $\alpha:[0, \infty) \rightarrow[0, \infty]$ be a nondecreasing function such that

$$
\lim _{t \downarrow 0} \alpha(t) / t=0 .
$$

2000 Mathematics Subject Classification: 46N10, 52A01.

Key words and phrases: Fréchet differentiability, strongly paraconvex functions, Asplund spaces. 
We say that $f$ is $\alpha(\cdot)$-paraconvex if there is a constant $C>0$ such that for all $x, y \in \Omega$ and $0 \leq t \leq 1$,

$$
f(t x+(1-t) y) \leq t f(x)+(1-t) f(y)+C \alpha(\|x-y\|) .
$$

For $\alpha(t)=t^{2}$ this definition was introduced in Rolewicz (1979a) and the $t^{2}$-paraconvex functions were called simply paraconvex. In Rolewicz (1979b) the notion was extended to the case of $\alpha(t)=t^{\gamma}, 1 \leq \gamma \leq 2$, and the $t^{\gamma_{-}}$ paraconvex functions were called $\gamma$-paraconvex.

Observe that the convex functions can be treated as 0-paraconvex functions.

We say that $f$ is strongly $\alpha(\cdot)$-paraconvex if there is a constant $C_{1}>0$ such that for all $x, y \in \Omega$ and $0 \leq t \leq 1$,

$$
f(t x+(1-t) y) \leq t f(x)+(1-t) f(y)+C_{1} \min \{t,(1-t)\} \alpha(\|x-y\|) .
$$

Obviously each strongly $\alpha(\cdot)$-paraconvex function is $\alpha(\cdot)$-paraconvex, but the converse is not true (Rolewicz (2000)).

The simplest examples of strongly $\alpha(\cdot)$-paraconvex functions are sums of convex and continuously differentiable functions, but the class of strongly $\alpha(\cdot)$-paraconvex functions is larger.

The notion of strongly $\alpha(\cdot)$-paraconvex functions can be treated as a uniformization of the notion of approximate convex functions introduced by Luc, Ngai and Théra (1999) (see Rolewicz (2001b)).

It is known that a convex function has a directional derivative at each point. The same holds for strongly $\alpha(\cdot)$-paraconvex functions.

Proposition 1.1. Let $\Omega$ be a convex subset of a Banach space $X$. Let $f: \Omega \rightarrow \mathbb{R}$ be a strongly $\alpha(\cdot)$-paraconvex function. Then at each $x \in \Omega$ the function $f$ has a directional derivative in any direction $h$ such that $x+h \in \Omega$.

Proof. For simplicity we set $\tilde{f}(t)=f\left(x_{0}+t h\right)-f\left(x_{0}\right)$. We shall show that $\lim _{t \downarrow 0} \widetilde{f}(t) / t$ exists.

The first step is to show that $\lim \sup _{t \downarrow 0} \widetilde{f}(t) / t$ is finite. Indeed, by strong $\alpha(\cdot)$-paraconvexity of $f$,

$$
\frac{\widetilde{f}(t)}{t} \leq \widetilde{f}(1)+C \frac{\alpha(t\|h\|)}{t} .
$$

Thus

$$
\limsup _{t \downarrow 0} \widetilde{f}(t) / t \leq \widetilde{f}(1) .
$$

This means that there are a real $a$ and a sequence $\left\{t_{n}\right\}$ tending to 0 such that

$$
\lim _{n \rightarrow \infty} \tilde{f}\left(t_{n}\right) / t_{n}=a .
$$


The next step is to show that the limits $\lim _{n \rightarrow \infty} \tilde{f}\left(t_{n}\right) / t_{n}$ are the same for all sequences tending to 0 .

Indeed, let $\tau_{m} \rightarrow 0$ and

$$
\lim _{t \downarrow 0} \tilde{f}\left(\tau_{m}\right) / \tau_{m}=b .
$$

Suppose that $\tau_{m}<t_{n}$. Then by strong $\alpha(\cdot)$-paraconvexity of $f$,

$$
\begin{aligned}
\frac{\tilde{f}\left(\tau_{m}\right)}{\tau_{m}} & \leq \frac{1}{\tau_{m}}\left(\frac{\tau_{m}}{t_{n}} \tilde{f}\left(t_{n}\right)+C \frac{\tau_{m}}{t_{n}}\left(1-\frac{\tau_{m}}{t_{n}}\right) \alpha\left(t_{n}\|h\|\right)\right) \\
& =\frac{\tilde{f}\left(t_{n}\right)}{t_{n}}+C\left(1-\frac{\tau_{m}}{t_{n}}\right) \frac{\alpha\left(t_{n}\|h\|\right)}{t_{n}} .
\end{aligned}
$$

Thus $b \leq a$. Reversing the roles of $\left\{t_{n}\right\}$ and $\left\{\tau_{m}\right\}$ we get $a \leq b$. Therefore $a=b$.

2. Uniform approximate subdifferentiability. The proof of the Asplund theorem in the classical case of convex functions consists of two parts:

(a) a convex function defined on an open set has a subgradient at each point,

(b) if a function $f$ has a subgradient at each point, then there is a set $A_{\mathrm{F}} \subset \Omega$ of the first category such $f$ is Fréchet differentiable at every point $x_{0} \in \Omega \backslash A_{\mathrm{F}}$.

In the classical situation the first part is so trivial that it is not observed at all. But now we are in a different situation. It is necessary to define "subgradients" and to show that a strongly $\alpha(\cdot)$-paraconvex function has a "subgradient" at each point.

The definition can be found in the papers of Fabian (1989), Ioffe (1983), (1984), (1986), (1989), (1990), (2000) and Mordukhovich (1980), (1988). Namely, a linear functional $x^{*} \in X^{*}$ will be called an approximate subgradient of $f$ at a point $x$ if

$$
\liminf _{h \rightarrow 0} \frac{(f(x+h)-f(x))-x^{*}(h)}{\|h\|} \geq 0 .
$$

The set of all approximate subgradients of $f$ at $x$ is called the approximate subdifferential of $f$ at $x$ and denoted by $\left.\partial f\right|_{x}$, as in the classical case. Thus $\left.\partial f\right|_{(\cdot)}$ is a multifunction mapping the domain of $\left.\partial f\right|_{(\cdot)}$ into $2^{X^{*}}$.

Observe that (2.1) holds if and only there is a non-negative non-decreasing function $\beta_{x}$ defined on $[0, \infty)$ and such that $\lim _{u \downarrow 0} \beta_{x}(u)=0$ and

$$
\frac{(f(x+h)-f(0))-x^{*}(h)}{\|h\|} \geq-\beta_{x}(\|h\|) .
$$


Indeed, the function

$$
\beta_{x}(s)=\sup _{\{h:\|h\| \leq s\}}\left|\frac{(f(x+h)-f(x))-x^{*}(h)}{\|h\|}\right|
$$

has the required property.

Putting $\alpha_{x}(u)=u \beta_{x}(u)$ we can rewrite $(2.2)$ in the form

$$
f(x+h)-f(x) \geq x^{*}(h)-\alpha_{x}(\|h\|) .
$$

Unfortunately $\beta_{x}$ (and hence $\alpha_{x}$ ) can be different at each point and we are not able to use this definition for the problem of differentiation on a residual set. This prompts an idea of uniformization of this notion.

Let, as before, $\alpha:[0, \infty) \rightarrow[0, \infty]$ be a non-decreasing function such that $\lim _{t \downarrow 0} \alpha(t) / t=0$.

Let $f$ be a real-valued function defined on an open subset $\Omega$ of a Banach space $X$. Let $x \in X$. A linear functional $x^{*} \in X^{*}$ such that

$$
f(x+h)-f(x) \geq x^{*}(h)-\alpha(\|h\|)
$$

is called a uniform approximate subgradient of $f$ at $x$ with modulus $\alpha(\cdot)$ (or briefly an $\alpha(\cdot)$-subgradient of $f$ at $x$ ). The set of all $\alpha(\cdot)$-subgradients of $f$ at $x$ will be called the $\alpha(\cdot)$-subdifferential of $f$ at $x$, and denoted by $\left.\partial_{\alpha} f\right|_{x}$.

We say that $f$ is $\alpha(\cdot)$-subdifferentiable if $\left.\partial_{\alpha} f\right|_{x} \neq \emptyset$ for all $x \in \Omega$.

In a similar way, we say that $x^{*} \in X^{*}$ is an $\alpha(\cdot)$-gradient of $f$ at $x$ if

$$
\left|f(x+h)-f(x)-x^{*}(h)\right| \leq \alpha(\|h\|) .
$$

By linearity of $x^{*}$ and property $(1.1)$ of $\alpha(\cdot)$ the $\alpha(\cdot)$-gradient is unique. The notion of $\alpha(\cdot)$-gradient can be considered as a uniformization of Fréchet gradients.

We say that $f$ is $\alpha(\cdot)$-differentiable if it has $\alpha(\cdot)$-gradients for all $x \in \Omega$.

\section{3. $\alpha(\cdot)$-subdifferentiability of strongly $\alpha(\cdot)$-paraconvex functions.} We shall show that every strongly $\alpha(\cdot)$-paraconvex function is $\alpha(\cdot)$-subdifferentiable.

In the case of convex functions on open convex sets, this is a trivial consequence of the Hahn-Banach theorem.

In the general case the proof is based on the following two propositions:

Proposition 3.1 (Rolewicz (2000)). Every strongly $\alpha(\cdot)$-paraconvex function is locally Lipschitzian.

Proposition 3.2 (Rolewicz (2001a)). The $\alpha(\cdot)$-subdifferential of each strongly $\alpha(\cdot)$-paraconvex function is equal to its Clarke subdifferential.

As a trivial consequence we obtain

Proposition 3.3 (Rolewicz (2002)). Every strongly $\alpha(\cdot)$-paraconvex function is $\alpha(\cdot)$-subdifferentiable. 
In the case of $X$ with separable dual (i.e. a separable Asplund space) we have

Theorem 3.4 (Rolewicz (2002)). Let $A$ be an open convex set in a separable Asplund space $X$. Let $f: A \rightarrow \mathbb{R}$ be $\alpha(\cdot)$-subdifferentiable. Then there is a residual set $\Omega \subset A$ such that $f$ is Fréchet differentiable at every point $x_{0} \in \Omega$.

4. Main results. As in the case of convex functions (see Phelps (1989)), we have

Proposition 4.1. Let $(X,\|\cdot\|)$ be a real Banach space. Let $f$ be a realvalued function defined on an open convex subset $\Omega \subset X$. Suppose that $x^{*}$ is an $\alpha(\cdot)$-subgradient of $f$ at $x \in \Omega$. Then $x^{*}$ is the Fréchet gradient of $f$ at $x$ if and only if for every $\varepsilon>0$ there is $\delta>0$ such that

$$
\frac{f(x+t y)+f(x-t y)-2 f(x)}{t}<\varepsilon
$$

for all $y \in X$ such that $\|y\|=1$ and $0<t<\delta$, in other words,

$$
\lim _{t \rightarrow 0} \sup _{\{y \in X:\|y\|=1\}} \frac{f(x+t y)+f(x-t y)-2 f(x)}{t}=0 .
$$

Proof. Necessity. If $x^{*}$ is the Fréchet gradient at $x$, then for every $\varepsilon>0$ there is $\delta>0$ such that

$$
f(x+t y)-f(x)-x^{*}(t y)<\frac{\varepsilon}{2} t
$$

for all $y \in X$ such that $\|y\|=1$ and $0<t<\delta$. Replacing $y$ by $-y$ we obtain

$$
f(x-t y)-f(x)+x^{*}(t y)<\frac{\varepsilon}{2} t .
$$

Adding (4.2) and (4.3) yields (4.1).

Sufficiency. By the property $(1.1)$ of $\alpha(\cdot)$, for every $\varepsilon>0$ there is $\delta_{1}>0$ such that

$$
f(x+t y)-f(x)-x^{*}(t y)>-\varepsilon t
$$

for all $y \in X$ such that $\|y\|=1$ and $0<t<\delta_{1}$.

Replacing $y$ by $-y$ and multiplying by -1 we get

$$
f(x)-f(x-t y)-x^{*}(t y)<\varepsilon t
$$

On the other hand, (4.1) implies that there is $\delta_{2}>0$ such that

$$
f(x+t y)-f(x)-x^{*}(t y)<\varepsilon t+f(x)-f(x-t y)-x^{*}(t y)
$$

for $0<t<\delta_{2}$. Thus for $0<t<\delta=\min \left\{\delta_{1}, \delta_{2}\right\}$ by (4.4) and (4.5) we get

$$
-\varepsilon t<f(x+t y)-f(x)-x^{*}(t y)<\varepsilon t+f(x)-f(x-t y)-x^{*}(t y)<2 \varepsilon t .
$$

The arbitrariness of $\varepsilon$ implies that $x^{*}$ is the Fréchet gradient of $f$ at $x$. 
If $f$ is strongly $\alpha(\cdot)$-paraconvex we can replace the requirement that (4.1) holds for $t$ small enough by the condition that such a $t$ exists, and we obtain

Proposition 4.2. Let $(X,\|\cdot\|)$ be a real Banach space and $f$ a strongly $\alpha(\cdot)$-paraconvex function defined on an open convex subset $\Omega \subset X$. The function $f$ is Fréchet differentiable at a point $x \in \Omega$ if and only if for every $\varepsilon>0$ there is $t_{\varepsilon}>0$ such that

$$
\frac{f\left(x+t_{\varepsilon} y\right)+f\left(x-t_{\varepsilon} y\right)-2 f(x)}{t_{\varepsilon}}<\varepsilon
$$

for all $y \in X$ such that $\|y\|=1$.

The proof is based on the following lemma:

Lemma 4.3. Let $(X,\|\cdot\|)$ be a real Banach space and $f$ a strongly $\alpha(\cdot)$ paraconvex function defined on an open convex subset $\Omega \subset X$. Then for $x \in \Omega$ and all $y \in X$ of norm one, $0<s<1$, and $t>0$ such that $x \pm t y \in \Omega$ we have

$$
\begin{aligned}
& \frac{f(x+s t y)+f(x-s t y)-2 f(x)}{s t} \\
& \leq \frac{f(x+t y)+f(x-t y)-2 f(x)}{t}+2 \frac{\alpha(t)}{t} .
\end{aligned}
$$

Proof. Since $f$ is strongly $\alpha(\cdot)$-paraconvex,

$$
\begin{aligned}
& f(x+s t y) \leq(1-s) f(x)+s f(x+t y)+s \alpha(t), \\
& f(x-s t y) \leq(1-s) f(x)+s f(x-t y)+s \alpha(t) .
\end{aligned}
$$

Adding (4.9) and (4.10) we get

$$
\begin{aligned}
f(x+s t y)+ & f(x-s t y) \\
\leq & 2(1-s) f(x)+s f(x+t y)+s f(x-t y)+2 s \alpha(t) .
\end{aligned}
$$

Thus

$$
\begin{aligned}
& f(x+s t y)+f(x-s t y)-2 f(x) \\
& \leq s[f(x+t y)+f(x-t y)-2 f(x)]+2 s \alpha(t) .
\end{aligned}
$$

Dividing (4.12) by $s t$ we get (4.8).

Proof of Proposition 4.2. The necessity is obvious: it follows from Proposition 4.1 and the fact that each strongly $\alpha(\cdot)$-paraconvex function is $\alpha(\cdot)$-subdifferentiable.

Let $t_{\varepsilon}>0$ be such that $t_{\varepsilon}+2 \alpha\left(t_{\varepsilon}\right) / t_{\varepsilon}<\varepsilon$. Then Lemma 4.3 and (4.8) yield (4.7).

Proposition 4.4. Let $(X,\|\cdot\|)$ be a real Banach space. Let $f$ be a strongly $\alpha(\cdot)$-paraconvex function defined on an open convex subset $\Omega \subset X$. 
Then the set $G$ (possibly empty) of points $x \in \Omega$ where $f$ is Fréchet differentiable is a $G_{\delta}$ set.

Proof. We set

$$
\begin{aligned}
& G_{m}^{\delta}(f)=\{x \in \Omega: \bar{B}(x, \delta) \subset \Omega, \\
&\left.\sup _{\{y \in X:\|y\|=1\}} \frac{f(x+\delta y)+f(x-\delta y)-2 f(x)}{\delta}<\frac{1}{m}\right\},
\end{aligned}
$$

where as usual $\bar{B}(x, \delta)=\{y \in X:\|y-x\| \leq \delta\}$ denotes the closed ball of radius $\delta$ with center at $x$. Let $G_{m}(f)=\bigcup_{\delta>0} G_{m}^{\delta}(f)$, i.e.

$$
G_{m}(f)=\left\{x \in \Omega: \text { } \inf _{\substack{\delta>0 \\ \bar{B}(x, \delta) \subset \Omega}} \sup _{\{y \in X:\|y\|=1\}} \frac{f(x+\delta y)+f(x-\delta y)-2 f(x)}{\delta}<\frac{1}{m}\right\} .
$$

We shall show that the sets $G_{m}^{\delta}(f)$ are open.

Take any $x \in G_{m}^{\delta}(f)$. Since $f$ is strongly $\alpha(\cdot)$-paraconvex, it is locally Lipschitz. Hence there are $\delta_{1}>0$ and $M>0$ such that $|f(u)-f(v)| \leq$ $M\|u-v\|$, provided $u, v \in \bar{B}\left(x, \delta_{1}\right)$. Without loss of generality we may assume that $\bar{B}\left(x, \delta_{1}\right) \subset \Omega$.

Since $x \in G_{m}^{\delta}(f)$, there is $r$ such that

$$
\sup _{\{y \in X:\|y\|=1\}} \frac{f(x+\delta y)+f(x-\delta y)-2 f(x)}{\delta} \leq r<\frac{1}{m} .
$$

Let $\delta_{2}>0$ be smaller than $\min \left\{\delta_{1}, \frac{\delta}{3 M}\left(\frac{1}{m}-r\right)\right\}$. Then for any $z \in$ $\bar{B}\left(x, \delta_{2}\right)$ and any $y$ of norm one we have

$$
\begin{aligned}
& \frac{f(z+\delta y)+f(z-\delta y)-2 f(z)}{\delta} \leq \frac{f(x+\delta y)+f(x-\delta y)-2 f(x)}{\delta} \\
&+\frac{|f(x+\delta y)-f(z+\delta y)|}{\delta}+\frac{|f(x-\delta y)-f(z-\delta y)|}{\delta}+\frac{|f(x)-f(z)|}{\delta} \\
& \leq r+3 M \delta_{2}<1 / m .
\end{aligned}
$$

Hence the set $G_{m}^{\delta}(f)$ is open. Therefore so is $G_{m}(f)=\bigcup_{\delta>0} G_{m}^{\delta}(f)$. By Propositions 4.1 and $4.2, G=\bigcap_{m} G_{m}(f)$. Hence $G$ is a $G_{\delta}$ set.

Corollary 4.5. Let $(X,\|\cdot\|)$ be a real Banach space and $f$ a strongly $\alpha(\cdot)$-paraconvex function defined on an open convex subset $\Omega \subset X$. Suppose that the set $G$ of points $x \in \Omega$ where $f$ is Fréchet differentiable is dense in $\Omega$. Then it is a residual set (i.e. its complement is of the first Baire category). 
Proof. Let $F=\Omega \backslash G$ and $F_{m}=\Omega \backslash G_{m}$. Since $G_{m}$ is open, $F_{m}$ is closed. Since $G$ is dense, so is $G_{m}$. Thus $F_{m}$ is nowhere dense and the set $F=\bigcup_{m} F_{m}$ is of the first Baire category.

TheOREm 4.6. Let $\Omega$ be an open convex set in an Asplund space $X$. Let $f$ be a strongly $\alpha(\cdot)$-paraconvex function defined on $\Omega$. Then the set $G$ of points where $f$ is Fréchet differentiable is a dense $G_{\delta}$ set (hence residual).

Proof. Suppose that $G$ is not dense in $\Omega$. We shall show that there is a separable subspace $E \subset X$ such that the set of points of Fréchet differentiability of the restriction $\left.f\right|_{\Omega \cap E}$ is not dense in $\Omega \cap E$.

We denote as before by $G_{m}(f)$ the set of those $x$ for which there is a $\delta>0$ such that $\bar{B}(x, \delta) \subset \Omega$ and

$$
\sup _{\{y:\|y\|=1\}} \frac{f(x+\delta y)+f(x-\delta y)-2 f(x)}{\delta}<\frac{1}{m} .
$$

By our assumption there are $m$ and an open set $U \subset \Omega$ such that $U \cap G_{m}(f)=\emptyset$. Thus for $x \in U$ and all $\delta>0$ such that $\bar{B}(x, \delta) \subset \Omega$,

$$
\sup _{\{y:\|y\|=1\}} \frac{f(x+\delta y)+f(x-\delta y)-2 f(x)}{\delta} \geq \frac{1}{m} .
$$

Take any $x_{1} \in U$. Take a decreasing sequence $\left\{\beta_{j}\right\}$ of positive numbers tending to 0 such that $\bar{B}\left(x_{1}, \beta_{1}\right) \subset \Omega$ and $\bar{B}\left(x_{1}, \beta_{1}\right) \cap G_{m}(f)=\emptyset$.

By (4.13) for $j$ large enough we can find an element $y_{1, j}$ of norm one such that

$$
\frac{f\left(x_{1}+\beta_{j} y_{1, j}\right)+f\left(x_{1}-\beta_{j} y_{1, j}\right)-2 f\left(x_{1}\right)}{\beta_{j}}>\frac{1}{2 m} .
$$

Take $\delta>0$ such that $\bar{B}\left(x, \delta_{1}\right) \subset \Omega$ and

$$
\frac{\alpha(\delta)}{\delta}>\frac{1}{2 m} \text {. }
$$

By Lemma 4.3 and (4.15) there is a constant $\varepsilon_{m}>0$ depending only on $m$ such that for $\delta \geq \beta_{j}$ such that $\bar{B}\left(x_{1}, \delta\right) \subset \Omega$ and

$$
\frac{f\left(x_{1}+\delta y_{1, j}\right)+f\left(x_{1}-\delta y_{1, j}\right)-2 f\left(x_{1}\right)}{\delta}>\varepsilon_{m} .
$$

Since $\beta_{j} \rightarrow 0$, for all $\delta>0$ such that $\bar{B}\left(x_{1}, \delta\right) \subset \Omega$ we have

$$
\sup _{j \geq 1} \frac{f\left(x_{1}+\delta y_{1, j}\right)+f\left(x_{1}-\delta y_{1, j}\right)-2 f\left(x_{1}\right)}{\delta}>\varepsilon_{m} .
$$

We denote by $E_{1}$ the closed linear span of the set $\left\{x_{1}, y_{1,1}, y_{1,2}, \ldots\right\}$. Of course $U \cap E_{1} \neq \emptyset$ and $E_{1}$ is separable. Now we construct by induction a sequence $\left\{E_{1}, E_{2}, \ldots\right\}$ of separable spaces such that $E_{k} \subset E_{k+1}$ and $U \cap E_{k} \neq \emptyset$. 
Suppose that we have constructed spaces $E_{1}, \ldots, E_{k}$. Let $\left\{x_{k, p}\right\}$ be dense in $U \cap E_{k}$. Then by (4.17) for each $x_{k, p}$ we can find a sequence $\left\{y_{p, j}\right\}$ of elements of norm one such that for all $p$ for sufficiently small $\delta$ (depending on $k$ and $p$ )

$$
\sup _{j \geq 1} \frac{f\left(x_{k, p}+\delta y_{p, j}\right)+f\left(x_{k, p}-\delta y_{p, j}\right)-2 f\left(x_{k, p}\right)}{\delta}>\varepsilon_{m} .
$$

We denote by $E_{k+1}$ the closed linear span of the set $\left\{x_{k, p}, y_{p, j}\right.$ : $p=1,2, \ldots, j=1,2, \ldots\}$. Clearly $U \cap E_{k+1} \neq \emptyset$ and $E_{k+1}$ is separable.

We put $E=\overline{\bigcup_{k} E_{k}}$. It is easy to see that the sequence $\left\{x_{k, p}\right\}, k, p=$ $1,2, \ldots$, is dense in $E$. By construction, it is easy to see that if $m_{1}$ is such that $1 / m_{1}<\varepsilon_{m}$, then the points $x_{k, p}, k, p=1,2, \ldots$, do not belong to $G_{m_{1}}\left(\left.f\right|_{E}\right)$. Since the set $G_{m_{1}}\left(\left.f\right|_{E}\right)$ is open in $E$ and disjoint from $E \cap U$ we conclude that $\left.f\right|_{E}$ is not differentiable at any point of the open set $E \cap U$.

This is a contradiction, since $\left.f\right|_{E}$ is a strongly $\alpha(\cdot)$-paraconvex function defined on an open set in the separable space $E$, and by Theorem 3.4 it is differentiable on a residual set.

\section{References}

E. Asplund (1966), Farthest points in reflexive locally uniformly rotund Banach spaces, Israel J. Math. 4, 213-216.

E. Asplund (1968), Fréchet differentiability of convex functions, Acta Math. 121, 31-47.

M. Fabian (1989), Subdifferentiability and trustworthiness in the light of a new variational principle of Borwein and Preiss, Acta Univ. Carolinae 30, 51-56.

A. D. Ioffe (1984), Approximate subdifferentials and applications I, Trans. Amer. Math. Soc. $281,389-416$.

A. D. Ioffe (1986), Approximate subdifferentials and applications II, Mathematika 33, 111-128.

A. D. Ioffe (1989), Approximate subdifferentials and applications III, ibid. 36, 1-38.

A. D. Ioffe (1990), Proximal analysis and approximate subdifferentials, J. London Math. Soc. $41,175-192$.

A. D. Ioffe (2000), Metric regularity and subdifferential calculus, Uspekhi Mat. Nauk 55, no. 3, 104-162 (in Russian).

A. Jofré, D. T. Luc and M. Théra (1998), $\varepsilon$-subdifferential and $\varepsilon$-monotonicity, Nonlinear Anal. 33, 71-90.

A. Jourani (1996), Subdifferentiability and subdifferential monotonicity of $\gamma$-paraconvex functions, Control Cybernet. 25, 721-737.

D. T. Luc, H. V. Ngai and M. Théra (1999), On $\varepsilon$-convexity and $\varepsilon$-monotonicity, in: Calculus of Variations and Differential Equations, A. Ioffe et al. (eds.), Res. Notes Math. 410, Chapman \& Hall, 82-100.

D. T. Luc, H. V. Ngai and M. Théra (2000), Approximate convex functions, J. Nonlinear Convex Anal. 1, 155-176.

S. Mazur (1933), Über konvexe Mengen in linearen normierten Räumen, Studia Math. 4, $70-84$. 
B. S. Mordukhovich (1980), Metric approximations and necessary optimality conditions for general classes of nonsmooth extremal problems, Soviet Math. Dokl. 22, 526-530.

B. S. Mordukhovich (1988), Approximation Methods in Problems of Optimization and Control, Nauka, Moscow (in Russian).

D. Pallaschke and S. Rolewicz (1997), Foundations of Mathematical Optimization, Math. Appl. 388, Kluwer, Dordrecht, 1997.

R. R. Phelps (1989), Convex Functions, Monotone Operators and Differentiability, Lecture Notes in Math. 1364, Springer.

D. Preiss and L. Zajíček (1984), Stronger estimates of smallness of sets of Fréchet nondifferentiability of convex functions, Rend. Circ. Mat. Palermo (2) Suppl. 3, 219-223.

R. T. Rockafellar (1970), Convex Analysis, Princeton Univ. Press.

R. T. Rockafellar (1980), Generalized directional derivatives and subgradient of nonconvex functions, Canad. J. Math. 32, 257-280.

S. Rolewicz (1979a), On paraconvex multifunctions, Oper. Res. Verfahren 31, 540-546.

S. Rolewicz (1979b), On $\gamma$-paraconvex multifunctions, Math. Japon. 24, 293-300.

S. Rolewicz (1981), On conditions warranting $\Phi_{2}$-subdifferentiability, Math. Programming Stud. 14, 215-224.

S. Rolewicz (1993), Generalization of Asplund inequalities on Lipschitz functions, Arch. Math. (Basel) 61, 484-488.

S. Rolewicz (1994), On an extension of Mazur's theorem on Lipschitz functions, ibid. 63, $535-540$.

S. Rolewicz (1999), On $\alpha(\cdot)$-monotone multifunctions and differentiability of $\gamma$-paraconvex functions, Studia Math. 133, 29-37.

S. Rolewicz (2000), On $\alpha(\cdot)$-paraconvex and strongly $\alpha(\cdot)$-paraconvex functions, Control Cybernet. 29, 367-377.

S. Rolewicz (2001a), On the coincidence of some subdifferentials in the class of $\alpha(\cdot)$ paraconvex functions, Optimization 50, 353-360.

S. Rolewicz (2001b), On uniformly approximate convex and strongly $\alpha(\cdot)$-paraconvex functions, Control Cybernet. 30, 323-330.

S. Rolewicz (2002), On $\alpha(\cdot)$-monotone multifunctions and differentiability of strongly $\alpha(\cdot)$ paraconvex functions, ibid. 31, 601-619.

Institute of Mathematics

Polish Academy of Sciences

Śniadeckich 8, P.O. Box 21

00-956 Warszawa, Poland

E-mail: rolewicz@impan.gov.pl

Received May 5, 2004

Revised version December 10, 2004 